\title{
Density of States of Landau Levels from Activated Transport and Capacitance Experiments
}

D. Weiss ${ }^{1 *}$, K.v. Klitzing ${ }^{1}$, and V. Mosser ${ }^{2+}$

${ }^{1}$ MPI für Festkörperforschung, D-7000 Stuttgart, Fed. Rep. of Germany

${ }^{2}$ Physik-Department, Technische Universität München,

D-8046 Garching, Fed. Rep. of Germany

Abstract. In this publication we demonstrate that a combination of capacitance measurements with an analysis of thermally activated conductivity seems to be useful for the determination of the density of states (DOS) of Landau levels in two-dimensional systems. The experimental results indicate that no real energy gap exists, but that a nearly energy-independent background density of states (which decreases with increasing electron mobility) is present. Close to the center of the Landau levels the DOS can be described by a Gaussian lineshape with a line width which increases proportional to the square root of the magnetic field. This result agrees with the prediction within the self-consistent Born approximation, but the expected variation $\Gamma \sim \sqrt{1 / \mu}$ of the line width $\Gamma$ with the mobility $\mu$ of the electrons could not be confirmed.

\section{Introduction}

A microscopic theory of the quantum Hall effect should give a correct description not only of the quantized resistivity values $\rho_{x y}=h / i e^{2}$ but also of the transitions between the plateaus and the values of the finite resistivity $\rho_{x x}$. Such transport calculations are extremely complicated since the theory itself is complicated, and in addition not enough information is available about the scattering centers. The published theories are based on certain approximations and assumptions about the distribution, the strength and the range of the scattering potential. A first test whether such assumptions are realistic should be available from a comparison between the calculated and the measured density of states $D(E)$, since calculations of $D(E)$ are much easier than a transport theory for $\rho_{x x}(B)$ which includes complicated phenomena like localization and correlation. One of the first theories of the density of states (DOS) assumed shortrange scatterers, which leads, within the self-consistent Born approximation, (SCBA) to a broadening of the discrete energy spectrum (expected for an ideal two-dimensional electron gas without scattering) into an elliptic lineshape for the DOS [1]. Higher order approximations show that an exponentially decaying $D O S$ is expected for energies $E-E_{n}$ larger than the linewidth of the Landau levels $E_{n}$ [2], so that a real energy gap with vanishing DOS may not be present, but the DOS at midpoint between two Landau levels should decrease drastically if the magnetic field (energy separa-

* Present address: Physik-Department, D-8046 Garching

+ New address: GIERS-Schl umberger, F-92124 Montrouge, France 

tion between adjacent Landau levels) is increased. Experimental information about the DOS can be obtained from measurements of the specific heat [3], from magnetization measurements [4], from temperature-dependent resistivity measurements in the regime of the Hall plateaus [5] or from capacitance measurements $[6,7]$. In this article we will discuss in more detail the derivation of the DOS from an analysis of the temperaturedependent resistivity (chapter 2) and from temperature-dependent capacitance measurements (chapter 3 ). The following discussion is based on a picture which does not include many-body effects. The notation "density of states (DOS)" in this paper is used to characterize the electronic properties within a single particle picture.

\section{Activated resistivity}

The measurements of the resistivity $\rho_{x x}$ in a strong magnetic field (Shubnikov-de Haas oscillations) were carried out on GaAs-AlGaAs heterostructures with mobilities $14,000<\mu<550,000 \mathrm{~cm}^{2} \mathrm{~V}^{-1} \mathrm{~s}^{-1}$ and carrier densities $1.4 \cdot 10^{11}<n_{s}<4.2 \cdot 10^{11} \mathrm{~cm}^{-2}$. The devices have Hall geometry with a typical length of about $3 \mathrm{~mm}$, a width of about $0.4 \mathrm{~mm}$ and a distance between potential probes of $0.5 \mathrm{~mm}$. The device current was kept below $1 \mu \mathrm{A}$ where electron heating is negligibly small.

The temperature dependence of $\rho_{x x}^{\min }$ (where $\rho_{x x}^{\min }$ means the minimum in the resistivity. which corresponds to a Fermi level position very close to the midpoint between two Landau levels) in the temperature range $2 K<T<20 K$ is usually dominated by an exponential term corresponding to

$$
\rho_{x x}^{\min } \sim \exp \left\{-\frac{E_{a_{2}} \max }{k T}\right\}
$$

where $E_{a, \max }$ denotes the measured activation energy. Measured activation energies, a $_{\text {a max }}$ for different samples at different magnetif field values are shown $i^{m} F_{i g .1}$. The filling factor $i$, defined as $i=n_{s} \cdot \frac{h}{e B}$ corresponds always to a fully occupied lowest Landau level ( $i=4$ for $(100)$ silicon MOSFETs and $i=2$ for GaAs-AlGaAs heterostructures). Since the measured activation energy $E_{a}$ max agrees fairly well with half of the cyclotron energy $\hbar_{\omega_{0}}$, this activation energy is interpreted as the energy difference between the Fermi energy $E_{F}$ and the center of the Landau level $E_{n}$. For the sake of simplicity we assume that the mobility edge of the Landau level is

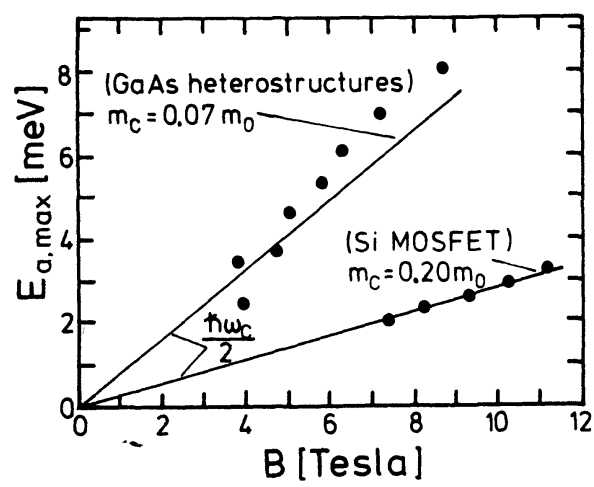

Fig. 1:

Measured activation energies $E_{\text {a max }}$ in the resistivity at a filfing factor corresponding to a fully occupied lowest Landau level as a function of the magnetic field $B$. The solid lines correspond to half of the cyclotron energy. 

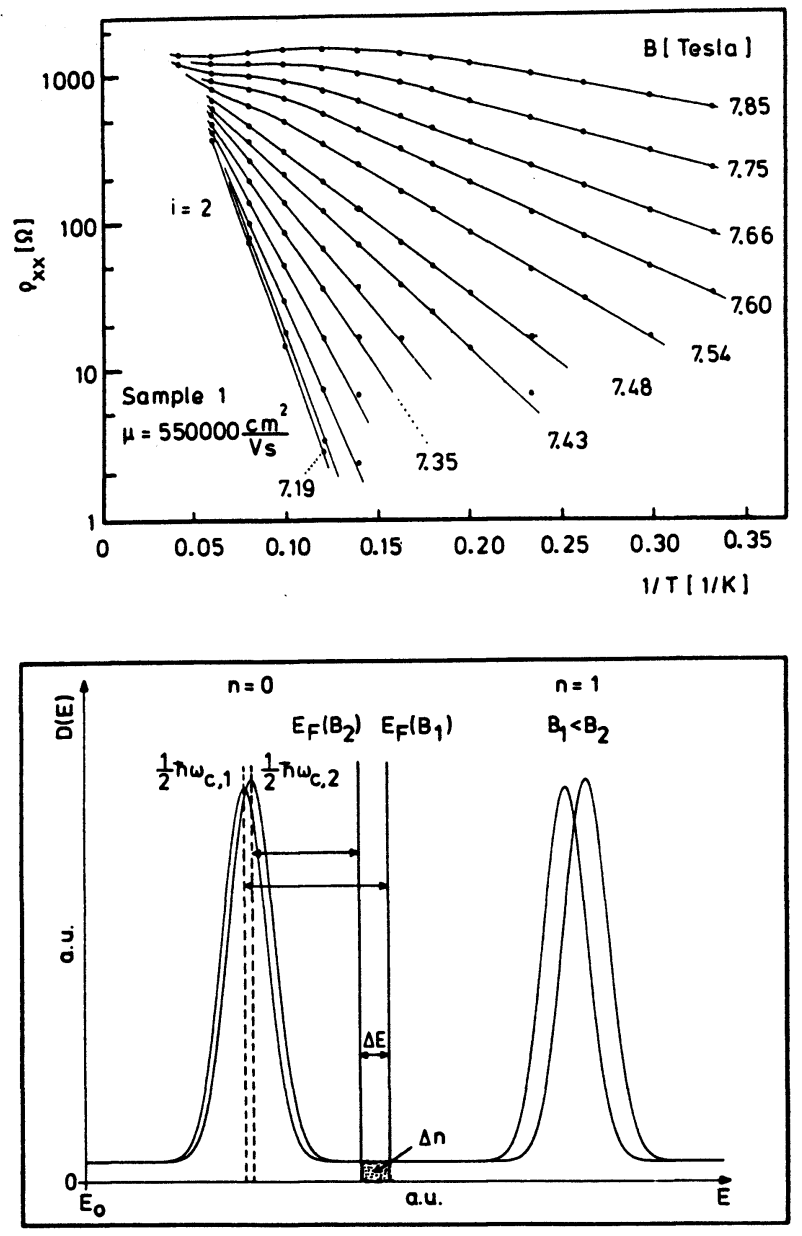

Fig. 2:

Temperature dependence of the resistivity $\rho_{x x}$ at different magnetic fields close to a filling factor $i=2$.
Fig. 3:

Model density of states at two different magnetic fields used to explain the reconstruction of the DOS from an analysis of the activation energies $E_{a}$ $\left(B_{i}\right)$.

located at the center of the Landau level, in agreement with calculations of the localization length [8] and percolation theories [9]. Furthermore the mobility edge should remain fixed, independent of the temperature and the carrier density. Changing the position of a Landau level $E_{n}$ relative to the Fermi energy $E_{F}$ (by changing the magnetic field) results in a reduced activation energy $E_{a}=\left|E_{n}-E_{F}\right|$. This motion of the Landau levels relative to the Fermi level if the filling factor of the Landau levels is varied is clearly visible in Fig.2. Since a filling factor change corresponds to a shift of the Fermi level, equivalent to a change $\Delta n$ in the carrier density at fixed magnetic field, a mean value for the density of states can be deduced. This is demonstrated in more detail in Fig.3. Shown is a model DOS at two different magnetic fields $B_{2}>B_{1}(T=0)$. If the magnetic field is raised from $B_{1}$ to $B_{2}$ the Fermi energy is shifted from $E_{F}\left(B_{1}\right)$ to the lower energy position $E_{F}\left(B_{2}\right)$ if the carrier density is kept constant. The variation of $\Delta E$ of the Fermi energy corresponds approximately (if the Fermi energy is located in the tails of the Landau levels between 
$n=0$ and $n=1$ ) to a change in the carrier density of

$$
\Delta n \approx \frac{e}{\operatorname{Th}}\left(B_{2}-B_{1}\right)
$$

Since the energy difference between the two Fermi level positions is given by

$$
\Delta E=E_{a}\left(B_{1}\right)-E_{a}\left(B_{2}\right)-\frac{1}{2} \hbar\left(\omega_{c, 2}-\omega_{c, 1}\right)
$$

the density of states can be deduced:

$$
D(E) \approx \frac{\Delta n}{\Delta E}
$$

It should be noted that the "point by point" construction of the DOS discussed in this chapter becomes incorrect in the energy region where the DOS changes drastically with energy. From the above it is clear that the change of the activation energy with magnetic field contains information about the density of states in the localized region between Landau levels. The change of the activation energy $E_{a}$ with magnetic field obtained from the $\rho_{x x}$-data shown in Fig.2 (sample 1, $\mu=550,000 \mathrm{~cm}^{2} \mathrm{v}^{-1} \mathrm{~s}^{-1}, \mathrm{n}_{\mathrm{s}}=3.5 \cdot 10^{11} \mathrm{~cm}^{-2}$ ) is plotted in Fig.4a. Using (1) to (3) we can deduce the density of states in the high-energy tail of the Landau level $n=0$ (Fig.4b). Figure $4 c$ shows the DOS of the lower mobility sample $3\left(\mu=180,000 \mathrm{~cm}^{2} \mathrm{~V}^{-1} \mathrm{~s}^{-1}, \mathrm{n}_{\mathrm{s}}=1.8 \cdot 10^{11} \mathrm{~cm}^{-2}\right)$ over the whole Hall plateau region $i=2$. The density of states $D(E)$ in Fig. $4 b, c$ is shown as a function of the energy relative to the center of the plateau region. The maximum of $D(E)$ close to $E=0$ is an artifact, since for the Fermi energy at $E=0$ two Landau levels contribute to the thermally activated conductivity, which complicates the analysis of the experimental data. If the Fermi energy is shifted out of the midpoint between two Landau levels by more

a.)

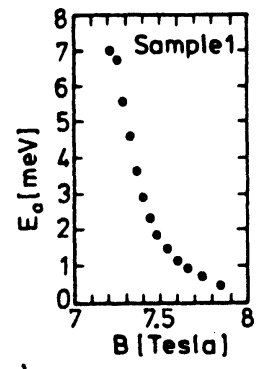

b.)

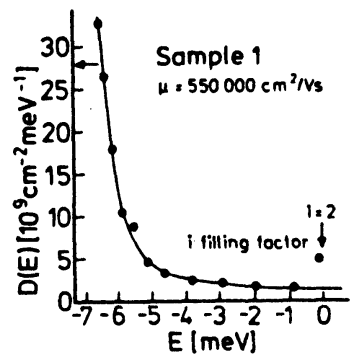

c.)

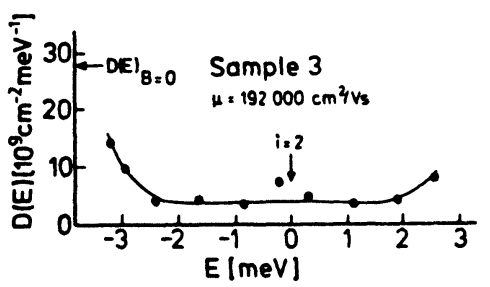

Fig. 4:

Change of the measured activation energy $E_{a}$ as a function of the magnetic field $B(a)$, and reconstructed DOS as a function of energy for sample $1(b)$ and $3(c)$. 
than the thermal energy $k T$, the contribution of the Landau level with the higher activation energy becomes unimportant. The experimental result that the density of states is constant within $50 \%$ of the energy between Landau levels is typical for all samples investigated. Measurements of the DOS at filling factors $i=2$ and $i=4$ for one and the same sample show that the minimal density of states $D_{U G}$ is nearly independent of the filling factor and therefore of the energy separation between Landau levels.

The ideas above are checked by computer simulations based on a model in which the temperature dependence of the resistivity in the plateau region is dominated by a term

$$
\rho \sim \rho_{0}(T) \sum_{n} \exp \left(-\left|\frac{E_{n}-E_{F}}{k T}\right|\right)
$$

where $\rho_{0}(T)$ is a temperature-dependent prefactor. The position of the Fermi level is determined by solving numerically the equation

$$
n_{S}=\int_{0}^{\infty} D(E) f\left(E-E_{F}\right) d E
$$

where $f\left(E-E_{F}\right)$ is the Fermi distribution function. The carrier density $n_{S}$ is assumed to be constant in the investigated temperature range. The model DOS $D(E)$ in (6) was chosen as a Gaussian-like density of states superimposed on a constant background density of states $D_{U G}$

$$
D(E)=A \cdot \frac{B}{\Gamma} \cdot \sum_{n} \exp \left\{-\frac{\left(E-\left(n+\frac{1}{2}\right) \hbar \omega_{C}\right)^{2}}{2 \Gamma^{2}}\right\}+D_{U G}
$$

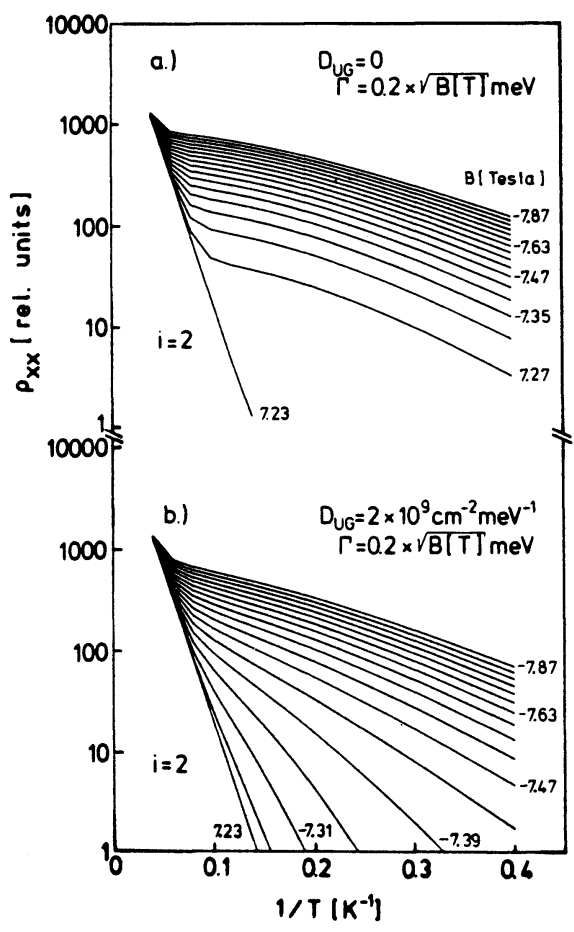

Fig. 5 :

Calculated resistivity $\rho_{x x}$ as a function of temperature âs suming different background densities $D_{\mu G}$. The carrier density used in the calculation is equal to the carrier density $n_{s}$ of sample 1 . 


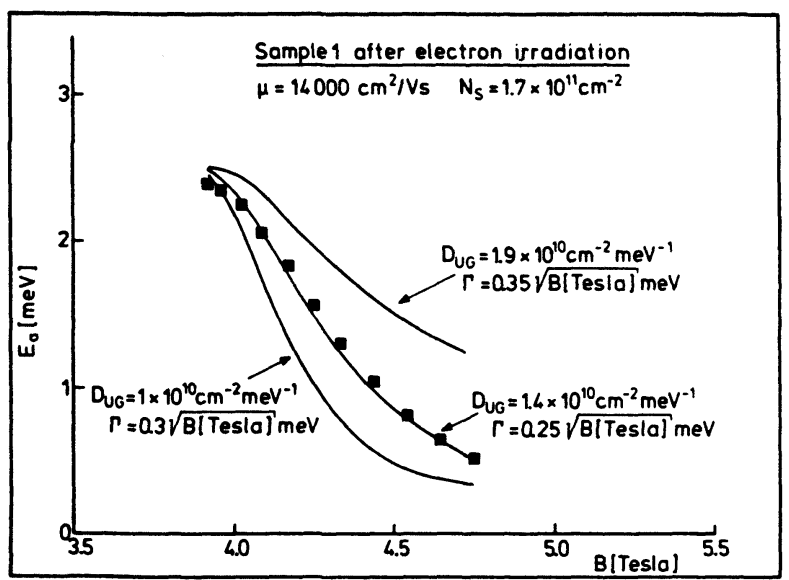

Fig. 6:

Measured activation energies of sample $1^{*}$ as a function of the magnetic field compared with activation energies deduced from calculated $\rho_{x x}$-curves using different combinations of background density of states $D_{U G}$ and Gaussian linewidth I.

where the constant $A$ is determined by the number of electrons in one Landau level and $r$ is the broadening parameter of the Gaussian distribution. Using (5) to (7) one can calculate temperature and magnetic fielddependent $\rho_{x x}$-values and deduce activation energies in analogy to Fig. 2. Calculations based on (5) to (7) demonstrate that the finite density of states $D_{U G}$ in the mobility gap influences strongly the result. Fig. 5 shows numerical calculations with and without using a background DOS

$D_{U G}=2 \cdot 10^{9} \mathrm{~cm}^{-2} \mathrm{meV}^{-1}$. The linewidth of the Gaussian in this calculation was $\Gamma=0.2 \cdot \sqrt{B[T]} \mathrm{meV}$. A reconstruction of the DOS by deducing activation energies from Fig.5b (the data used in the simulation correspond to sample 1 in Fig.2) in the temperature range of the experiments demonstrates that the constant background DOS is reproduced within $20 \%$.

Figure 6 summarizes the data obtained for a heterostructure (sample $1^{*}$ ) with a mobility of only $14,000 \mathrm{~cm}^{2} \mathrm{~V}^{-1} \mathrm{~s}^{-1}$ at $4.2 \mathrm{~K}$. This low mobility is achieved by irradiating sample 1 with $1 \mathrm{MeV}$ electrons. The experimentally deduced activation energies are compared to "calculated" ones - using different combinations of Gaussian linewidth $\Gamma$ and background DOS DUG - as a function of the magnetic field. The temperature-dependent prefactor was chosen as $\rho_{0}(T)=$ const $/ T$. The experimental data are best described by a Gaussian linewidth $\Gamma=0.25 \cdot \sqrt{B \mid T T}$ meV and a constant background $D_{U G}=1.4 \cdot 10^{10} \mathrm{~cm}^{-2} \mathrm{meV}^{-1}$.

Annealing of this electron-irradiated sample at $220^{\circ} \mathrm{C}$ leads to an increased mobility $\mu=28,000 \mathrm{~cm}^{2} \mathrm{~V}^{-1} \mathrm{~s}^{-1}$ (sample $1^{\star \star}$ ). The measured activation energy versus magnetic field is shown in Fig.7a. The best fit is obtained using a prefactor $\rho_{0}(T)=$ const, a Gaussian linewidth $\Gamma=0.25 \cdot \sqrt{B[T]}$ meV and $a$ constant background DOS $D_{U G}=9 \cdot 10^{9} \mathrm{~cm}^{-2} \mathrm{meV}^{-1}$. The use of the prefactor $\rho_{0}(T)$ looks somewhat arbitrary, but in fact the prefactor does not change remarkably the slope of $E_{a}(B)$, and therefore the value of the constant background density of states $D_{U G}$. This has been checked for prefactors const/T, const and const. T. This is shown in Fig. $7 \mathrm{~b}$ where the constant background DOS is reproduced within $20 \%$ independent of the prefactor $\rho_{0}(T)$. The determination of the linewidth $\Gamma$ however depends on the knowledge of the energy difference between mobility edge and Fermi level, and therefore on the absolute value of the activation energy. For this reason there remains some uncertainty in the determination of the linewidth $\Gamma$ by 

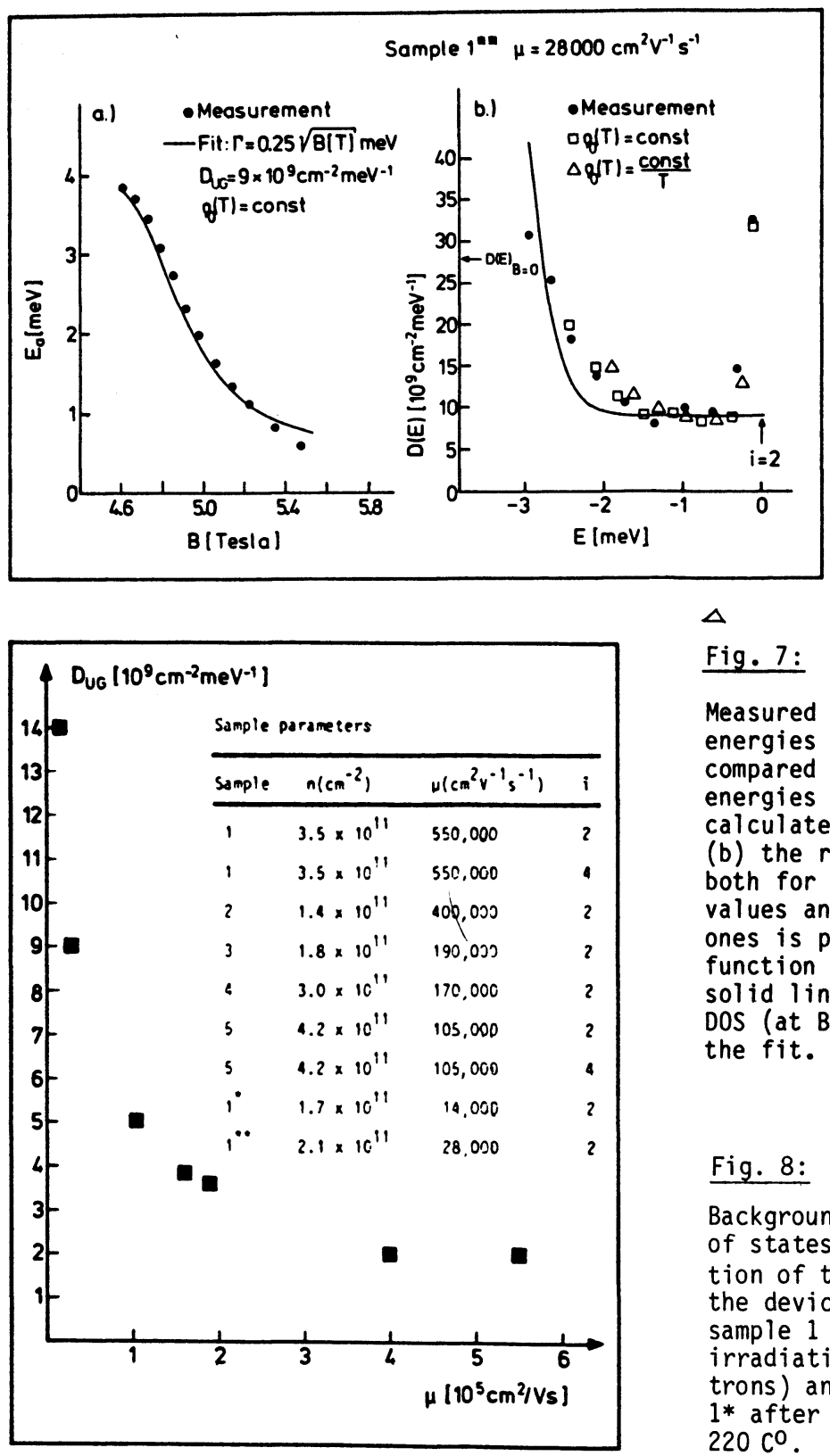

Fig. 7:

Measured activation energies of sample 1** compared with activation energies deduced from calculated curves (a). In (b) the reconstructed DOS both for measured $E_{a}(B)$ values and "calculated" ones is plotted as a function of energy. The solid line shows the model DOS (at $B=4.6 \mathrm{~T}$ ) used in the fit.

\section{Fig. 8:}

Background density of states DUG as a function of the mobility of the devices. 1* denotes sample 1 after electron irradiation ( $1 \mathrm{MeV}$ electrons) and $1 * *$ is sample $1 *$ after annealing at $220 \mathrm{co}^{\circ}$.

this method. The solid line in Fig.7b corresponds to the model DOS (filling factor $i=2$ ) used in these model calculations. The results of activated resistivity measurements are summarized in Fig. 8 where the constant background $D_{U G}$ is plotted as a function of the mobility. Also included is a table containing the parameters of the investigated samples. It should be 
noted that the reconstruction of the density of states from activated resistivity measurements is restricted to the tails of the Landau levels. Furthermore the $\sqrt{B}$ dependence of the linewidth $\Gamma$ assumed above cannot be proved with these measurements. Temperature-dependent measurements of the magnetocapacitance can overcome this problem and are discussed in the next section.

\section{Magnetocapacitance}

The capacitance experiments were carried out on gated GaAs-AIGaAs heterostructures with a Hall geometry. The mobilities of the samples were $220,000 \mathrm{~cm}^{2} \mathrm{~V}^{-1} \mathrm{~s}^{-1}$ and $800,000 \mathrm{~cm}^{2} \mathrm{~V}^{-1} \mathrm{~s}^{-1}$. The corresponding carrier densities were $2.25 \cdot 10^{11} \mathrm{~cm}^{-2}$ and $2.50 \cdot 10^{11} \mathrm{~cm}^{-2}$. For capacitance measurements all the Hall contacts were short-circuited and acted as a channel contact.

The signal was obtained by measuring the voltage drops at the sample and at a high-precision Boonton capacitance decade. This arrangement allows both a precise determination of the phase and the absolute value of the signal (see Fig.9b). The frequency chosen for the measurements was $223 \mathrm{~Hz}$. Measurements between $22.3 \mathrm{~Hz}$ and $446 \mathrm{~Hz}$ showed no change in the signal. The modulation amplitude was $5 \mathrm{mV}$, which corresponds to a modulation of $\Delta n \approx 4 \times 10^{9} \mathrm{~cm}^{-2}$. Further reduction of this amplitude showed no change in the signal. At each temperature the real part of the signal was monitored, and we checked that the signal was always purely capacitive for $C>C(B=0)$, even in the case of very low temperatures ( $T=1.64 \mathrm{~K})$ and high magnetic fields. Warming up and cooling down of the sample introduced no change in the signal.

The capacitance of a system, consisting of a metal-insulator-(with ionized impurities) semiconductor-sandwich (e.g. Au-AlGaAs-GaAs-heterostructure), depends not only on the thickness of the insulator but also on the DOS at the semiconductor side and on parameters of the material. Fig.9a

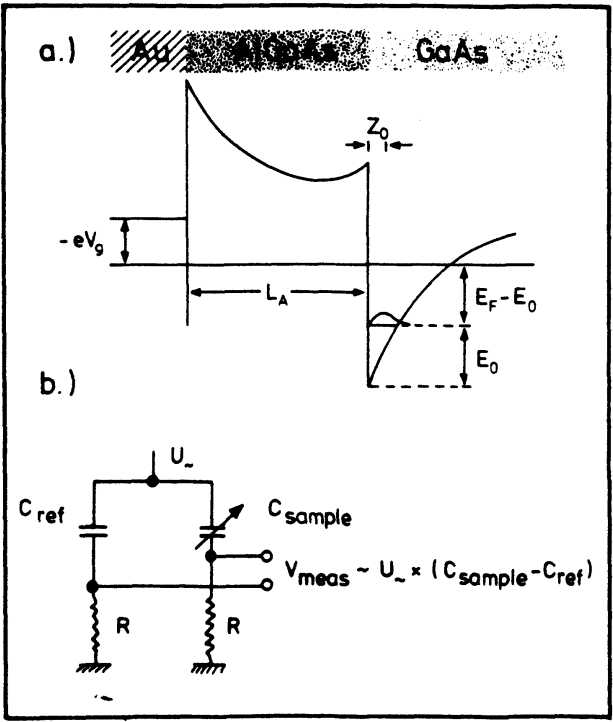

Fig. 9:

Schematic diagram of the conduction band edge (a) for a gated GaAs-AlGaAs heterostructure showing the quantities used in the derivations and schematic experimental set up (b). $U_{\sim}$ is the $A C$ component of the applied voltage with an amplitude of $5 \mathrm{mV}$. 
shows the band diagram of a heterostructure including a Schottky gate in contact with the AlGaAs. If the two depletion layers interpenetrate each other the gate voltage $V_{g}$ is connected to the carrier density $n_{s}$ by [10]

$$
v_{g}=\frac{e L_{A}}{\varepsilon_{i}} n_{s}+\frac{1}{e}\left[E_{0}+\left(E_{F}-E_{0}\right)\right]+K
$$

where $L_{A}$ is the thickness of the AlGaAs layer, $\varepsilon_{j}$ is the dielectric constant of the "insulator" and $K$ takes into account fixed charges in the AlGaAs and barrier heights at both interfaces. At low temperatures, carriers in the bulk of both materials are frozen out, so that $k$ is a constant. Differentiating (8) with respect to $n_{s}$ within the variational approximations of Stern [11], which take into account the $n_{s}$ dependence of the subband edge $E_{0}$, one obtains for the capacitance:

$$
\frac{1}{C}=\frac{1}{C_{A}}+\frac{\gamma z_{0}}{\varepsilon_{S}}+\frac{1}{e^{2} \frac{d n_{S}}{d\left(E_{F}-E_{0}\right)}}
$$

where $C$ is the measured differential capacitance at a given magnetic field, $C_{A}$ is the capacitance of the insulating AlGaAs layer, $\varepsilon_{s}$ is the dielectric constant of GaAs, $z_{0}$ is the average position of the electrons in the channel, $\gamma$ is a constant numerical factor between 0.5 and 0.7 , and $\mathrm{dn}_{\mathrm{S}} / \mathrm{d}\left(\mathrm{E}_{\mathrm{F}}-\mathrm{E}_{0}\right)$ is the thermodynamic $D O S$ at the Fermi level, in the following denoted as $\mathrm{dn}_{S} / \mathrm{dE}_{\mathrm{F}}$. The first two terms on the right-hand side of $(9)$ are assumed to be constant in a magnetic field, and thus changes of the capacitance are directly related to changes in the thermodynamic DOS of the 2DEG. At $T=0$ the total inverse capacitance in a magnetic field can be expressed as

$$
\frac{1}{C}=\frac{1}{C_{0}}-\frac{1}{e^{2} D_{0}}+\frac{1}{e^{2} D}
$$

where $C_{0}$ denotes the value of the total capacitance at $B=0, D$ is the DOS at the Fermi level in the presence of a magnetic field and $D_{0}$ is the DOS within the lowest subband, equal to $2.9 \times 10^{10} \mathrm{~cm}^{-2} \mathrm{meV}^{-1}$ in the absence of a magnetic field. At finite temperatures $D$ has to be replaced by $d_{s} / d E_{F}$.

The experimental results were compared with calculations of $C(B)$ assuming a Gaussian-like density of states superimposed on a constant background DOS DUG according to (7). The position of the Fermi level is determined again by solving numerically (6) and then

$$
\frac{d n_{S}}{d E_{F}}=\int_{0}^{\infty} D(E) \frac{d f\left(E-E_{F}\right)}{d E_{F}} d E
$$

is calculated numerically. With the temperature-dependent form of (10) and (11) one obtains $C(B)$. Spin splitting, which is small compared to the cyclotron energy for GaAs, is neglected in the calculations.

Some further considerations are necessary to fit the data using the expressions above. The minima and maxima of the measured capacitance are connected to minima and maxima in the DOS in the two-dimensional electron gas. A minimum in capacitance is obtained when the Fermi level is between two Landau levels. Additional calculations, assuming a Gaussian distribution of the electron density $n_{s}$, show that inhomogeneities strongly 


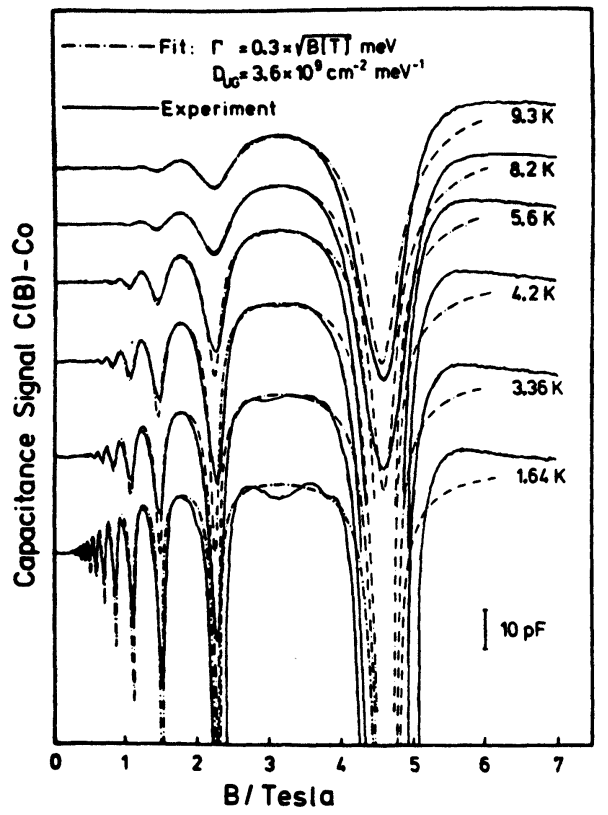

Fig.10:

Measured magnetocapacitance of a sample with a mobility of $220,000 \mathrm{~cm}^{2} \mathrm{~V}^{-1} \mathrm{~s}^{-1}$ (same material as sample 3 ) and corresponding fit using our model DOS with the parameters given in the plot. For the sake of clarity the curves are shifted vertically.

influence the minima but not the maxima of the capacitance at sufficient high magnetic fields. Furthermore, at low temperatures and high magnetic fields the capacitance signal is no longer purely capacitive, if the Fermi level position is between two Landau levels $\left(\sigma_{x x} \simeq 0\right)$. For this reason, it is advis-able to concentrate on the maxima of the measured capacitance to fit the data.

Figure 10 shows the capacitance data at different temperatures. Al.so shown is the fit to these data assuming a linewidth $\Gamma=0.3 \sqrt{B[T]}[\mathrm{meV}]$ and a background DOS of $3.6 \times 10^{9} \mathrm{~cm}^{-2} \mathrm{meV}^{-1}$. The value of the constant background is obtained from the temperature-dependent resistivity measurements in the regime of the Hall plateaus carried out on the same material (sample 3, see Fig.4c). At all investigated temperatures the calculated maxima of the magnetocapacitance are in excellent agreement with the experimental ones in the magnetic field range up to 5 Tesla. In Fig.1la calculated magnetocapacitance data using a $\sqrt{B}$ dependent Gaussian linewidth in the model DOS are compared to those assuming a magnetic field independent linewidth. Assuming a constant linewidth $\Gamma=0.54 \mathrm{meV}$ the magnetocapacitance for the Landau level $n=1$ is fitted correctly, but for higher Landau levels the resulting capacitance maxima are too small. On the other hand a linewidth $\Gamma=0.34 \mathrm{meV}$ describes correctly the capacitance for the Landau level $n=3$ but results in larger magnetocapacitance maxima for lower Landau levels.

The solid lines in Fig.11 correspond to the model calculation used to fit the data shown in Fig.10. Therefore a fit of the experimental data with a magnetic field independent linewidth as well as a linewidth which differs from the assumed value by more than $10 \%$ was not possible. It should be mentioned that the assumption of a vanishing background $D_{U G}$ broadens $\Gamma$ only by about $10 \%$. The depths of the measured capacitance minima at fow temperatures are smaller than the calculated ones as long as 

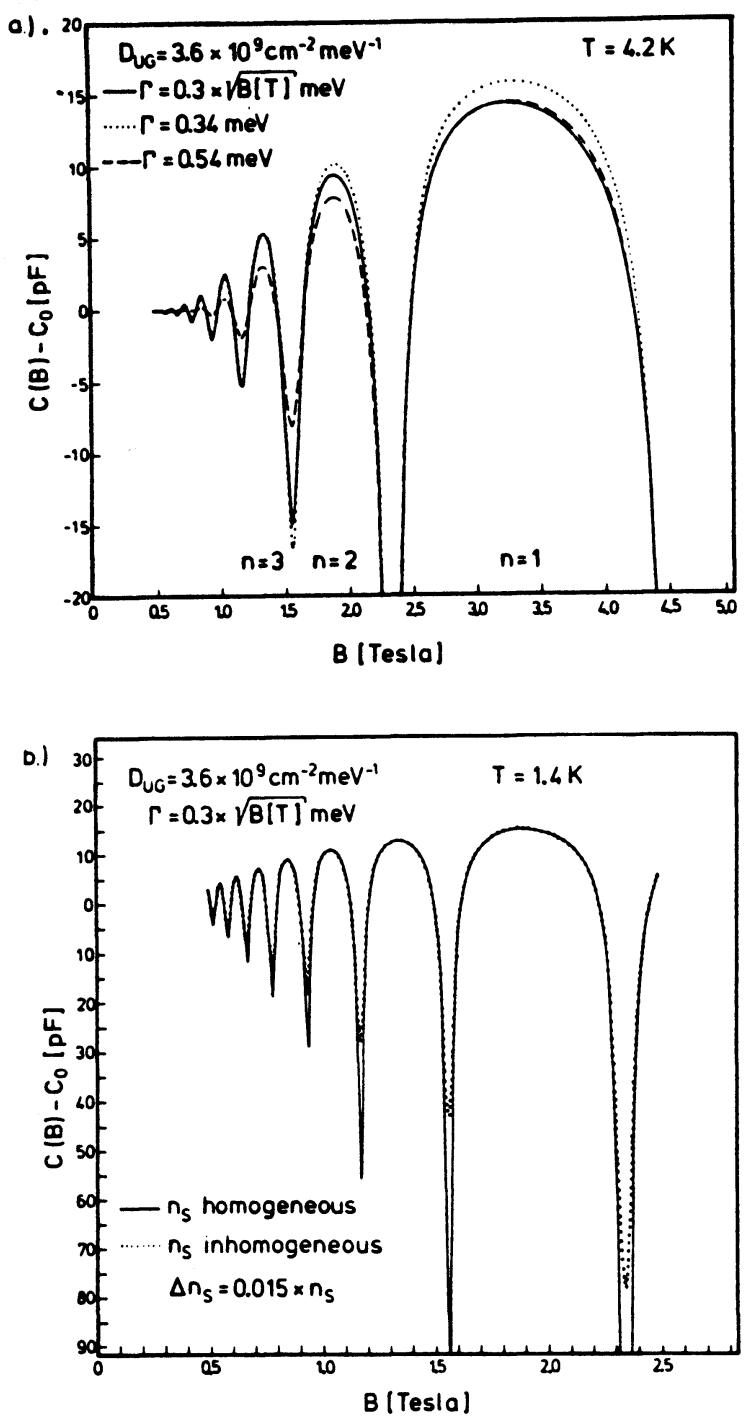

Fig. 11:

(a) Magnetocapacitance obtained numerically for different broadening parameters $\Gamma$. (b) shows the influence of inhomogeneities - assumed as a Gaussian distribution of the carrier density $n_{S}$ - on capacitance minima and maxima. The solid lines in (a) and correspond to the model calculation used to fit the data shown in Fig. 10.

resistivity effects in the channel are negligible (low B-field). This is attributed to inhomogeneities. Their influence is demonstrated in Fig.11b. The assumption of a Gaussian distribution of the carrier density $n_{5}$ with a broadening parameter $\Delta n_{s}=0.015 \cdot n_{s}$ leads at $1.4 K$ to a remarkable reduction of the depth of the capacitance minima compared to the homogeneous case, but the maxima in the magnetocapacitance remain unchanged. The influence of the inhomogeneities decreases with increasing temperature. At higher magnetic fields the capacitance signal at the minima is governed by the small conductivity $\sigma_{x x}$ which becomes less important at higher temperatures. Therefore the $x i t$ works well for minima and maxima of magnetocapacitance at higher temperatures. The difference between experiment and cal- 


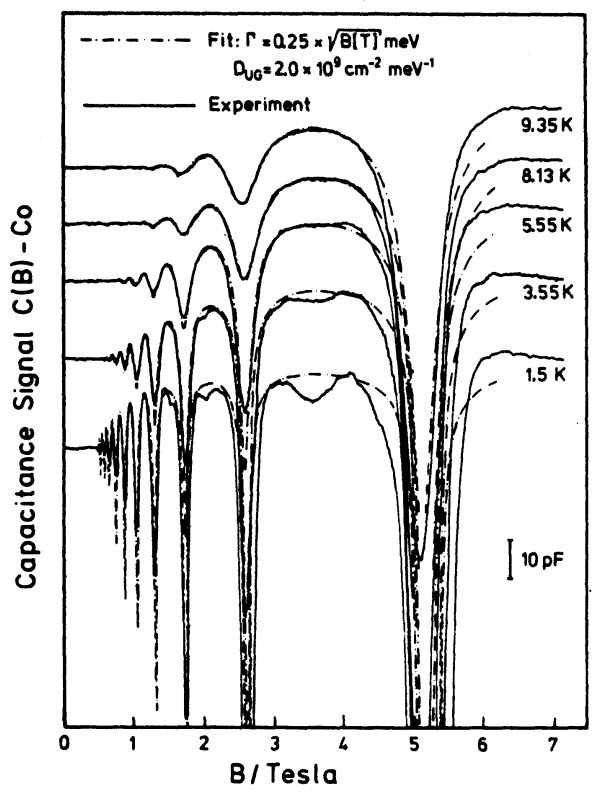

Fig.12:

Measured magnetocapacitance of a sample with a mobility of 800,000 $\mathrm{cm}^{2} \mathrm{~V}^{-1} \mathrm{~s}^{-1}$ and corresponding fit using our model DOS with the parameters given in the plot. For the sake of clarity the curves are shifted vertically.

culation if only one Landau level is filled cannot be explained yet. In Fig.12 the temperature-dependent magnetocapacitance data for a high-mobility sample $\left(\mu=800,000 \mathrm{~cm}^{2} v^{-1} \mathrm{~s}^{-1}, n_{\mathrm{s}}=2.50 \cdot 10^{11} \mathrm{~cm}^{-2}\right)$ are shown. The data are fitted with our model DOS using a Gaussian linewidth $\Gamma=0.25 \cdot \sqrt{B[T]}$ meV and a constant background DOS $D_{U G}=2 \cdot 10^{9} \mathrm{~cm}^{-2} \mathrm{meV}^{-1}$ according to Fig.8.

It should be emphasized that the constant background $D_{U G}$ is not necessary to fit the capacitance data, since the reduction of the measured capacitance minima compared to the calculated ones can be explained by inhomogeneities. On the other hand it is not possible to exclude the existence of a constant background density of states from our capacitance experiments.

Our model used to calculate numerically the magnetocapacitance requires some supplementary remarks. Actually not the carrier density $n_{s}$ but the Fermi level is kept constant during capacitance experiments. Using the notation of Fig.9a this means that the gate voltage $V_{g}$ is kept constant. Varying the magnetic field $B$ then leads to oscillations of the surface potential (bottom of the potential well) and to a charge transfer between gate and channel of the heterostructure. Since the amount of transferred charge is small compared to the two-dimensional carrier density $n_{s}$ the subband edge (taken relative to the bottom of the potential well) is' assumed to be constant. Equation ( 8 ) can then be rewritten as

$$
n_{s}+\frac{C_{A}}{e^{2}}\left(E_{F}-E_{0}\right)=\text { const }
$$

Combining (12) with (6) and taking all energies relative to the subband edge $E_{0}^{-}$leads to 


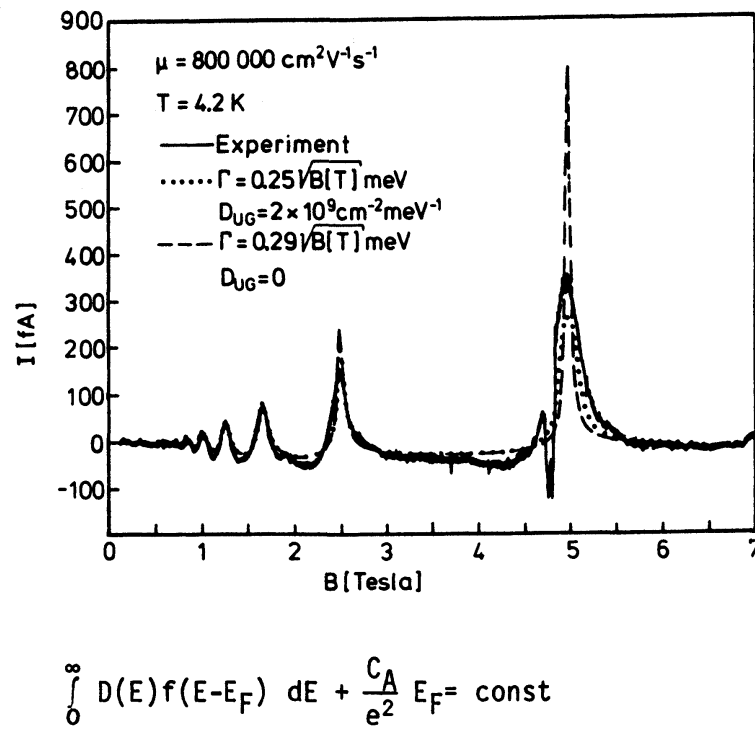

Fig.13:

Measured current flow between gate and channel as a function of the magnetic field. The fit is based on our model DOS (6) using the parameters shown in the plot.

where the constant can be determined at $B=0$. Equation (13) has to be solved numerically to give the correct position of the subband edge relative to Fermi level, and then the magnetocapacitance can be calculated using (11) and the temperature-dependent form of (10). Calculating the magnetocapacitance in the way described above results in a broadening of the width of the capacitance minima compared to calculations assuming a constant carrier density $n_{s}$. The difference, however, is small, and cannot be resolved in Fig.10 or Fig. 12 . The charge flow mentioned above can be determined by measuring the current between gate and channel as a function of the magnetic field $B$. The current flow is given by

$$
I(B)=A \cdot e \cdot \frac{d n_{s}}{d t}=A \cdot e \frac{d n_{s}}{d B} \cdot \frac{d B}{d t}
$$

where $A$ is the area of the two-dimensional electron gas and $d B / d t$ the sweep rate of the magnetic field. $d n_{s} / d B$ can be determined by solving (13) at different magnetic fields since the first term on the left-hand side is equal to the carrier density $n_{s}$. Therefore measurements of the current flow between gate and channel contain information about the density of states and may be a method to obtain new information about the density of states. Preliminary results are shown in Fig.13 where the current flow between gate and channel is plotted as a function of the magnetic field. This measurement was carried out on the high-mobility sample $\left(\mu=800,000 \mathrm{~cm}^{2} \mathrm{v}^{-1} \mathrm{~s}^{1}\right)$. The experimental data are compared with numerical calculations using our model DOS with the same parameters as shown in Fig.12. An additional calculation assuming a vanishing background $D_{U G}=0$ but a somewhat broadened linewidth $\Gamma=0.29 \cdot \sqrt{B[T]}$ meV is shown, too. It should be mentioned again that both sets of parameters fit the capacitance data shown in Fig.12.

\section{Summary}

The discussion above has shown that temperature-dependent resistivity measurements and capacitance measurements are complementary methods for the experimental determination of the DOS since the analysis of the acti- 
vated resistivity is restricted to the tails of the Landau levels, whereas magnetocapacitance experiments give information about the DOS close to the center of Landau levels. The analysis of the activated resistivity leads to the result of a constant or weak magnetic field-dependent background density of states whose magnitude increases if the mobility of the sample decreases. Measurements of the magnetocapacitance are compatible with the assumption of a background DOS but give no evidence for its existence, since the differences between experiment and calculation are well described by the influence of inhomogeneities. Inhomogeneities may also influence the results of heat capacity [3] and magnetization measurements $[4]$. Furthermore magnetocapacitance measurements demonstrate the $\sqrt{B}$ dependence of the Gaussian linewidth assumed. The linewidth $\Gamma$ however is larger than expected from the selfconsistent Born approximation (SCBA) assuming short-range scatterers, and does not follow the mobility dependence $\Gamma \sim \sqrt{1 / \mu}[1]$.

Further information about the density of states, especially about the background $D_{U G}$ may be obtained by analyzing carefully the current flow between gate and channel as a function of the magnetic field and temperature.

\section{References}

1. T. Ando, Y. Uemura, J.Phys.Soc.Jap. 36, 959 (1974)

2. R.R. Gerhardts, Surf.Sci. 58, 227 (1 $\overline{976}$ )

3. E. Gornik, R. Lassnig, G. Strasser, H.L. Störmer, A.C. Gossard, W. Wiegmann, Phys.Rev.Lett. 54, 1820 (1985)

4. J.P. Eisenstein, H.L. Störmer, V. Narayanamurti, A.Y. Cho, A.C. Gossard, Phys.Rev.Lett. 55, 875 (1985)

5. E. Stah1, D. Weiss, G. Weimann, K. V.Klitzing, K. Ploog, J.Phys. C18, L783 (1985)

6. T.P. Smith, B.B. Goldberg, P.J. Stiles, M. Heiblum, Phys.Rev. B32, 2696 (1985)

7. V. Mosser, D. Weiss, K. V.Klitzing, K. Ploog, G. Weimann, Solid State Commun., to be published

8. T. Ando, J.Phys.Soc.Jap. 53, 3101 (1984)

9. S.A. Trugman, Phys.Rev. B27, 7539 (1983)

10. D. Delagebeaudeuf, N.T. Linh, IEEE Trans. ED-29, 955 (1982)

11. F. Stern, Phys.Rev. B5, 4891 (1972) 